\title{
Nitrothiophenes as electrophiles in polar Diels-Alder reactions. Solvent effect and microwave irradiation
}

\author{
Anna F. Lopez Baena, Claudia D. Della Rosa, Pedro M.E. Mancini \\ Área Química Orgánica- Departamento de Química- Facultad de Ingeniería Química, Universidad Nacional del \\ Litoral. Santiago del Estero 2829. (3000) Santa Fe, Argentina. \\ FAX: +54-342-4571162. *e-mail:pmancini@fiq.unl.edu.ar
}

\begin{abstract}
In this work the electrophilic behaviour of nitrothiophenes in polar Diels-Alder toward dienes of different nucleophilicity is studied. In thermal conditions the reactions were developed in molecular solvents and in protic ionic liquids trying to compare the solvent effects on this class of reactions. In the presence of these solvents the reaction system was exposed to microwave irradiation as a complement of reaction conditions.
\end{abstract}

Keywords: nitrothiophene, Diels-Alder, nucleophilicity.

\section{Introduction}

The Diels-Alder (DA) reaction is one of the most useful processes in preparative organic chemistry. Its potential in heterocyclic chemistry and natural products synthesis is very well known. It provides the chemist with one of his best tools for the preparation of cyclic compounds having a six-membered ring. The process is in one step inter or intramolecular from a diene and a dienophile bearing an almost unlimited number of variants. It is worth noting that these variants exist not only in the substitution of the reaction component but also in the electronic nature of these dienes and dienophiles.

Benzothiophenes are an important class of heterocycles used as starting materials for the synthesis of bioactive structures and are present in a variety of pharmaceuticals substances Consequently, a considerable effort has been devoted to the development of efficient methods for the construction of these ring system ${ }^{1,2,3}$.

In the 1980s, studies of aromatic heterocycles such as indoles, benzofurans, pyrroles, furans, and thiophenes part of DA reactions demonstrate the viability of these systems as dienophiles. ${ }^{4}$ 
More recently we have confirmed that aromatic nitroheterocyclic act as electrophiles in DA reactions. $5,6,7,8$

Herein we report our findings electrophilic behaviour of nitrothiophenes when they were exposed to different dienes strongly, moderately and poorly activated, under thermal conditions, using molecular solvents and ionic liquids and in some experiences combining it with microwave irradiation.

\section{Results and discussion}

This research was carried out using 2-nitrothiophene 1 as dienophile. Isoprene 2, 1 trimethylsilyloxy-1,3-butadiene 3 and 1-methoxy-3-trimethylsilyloxy-1,3-butadiene (Danishefsky's diene) 4 were chosen as the dienes partners (Scheme 1).

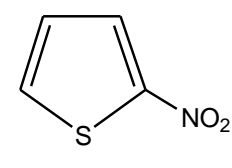

1

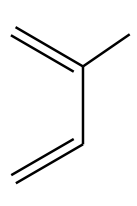

2

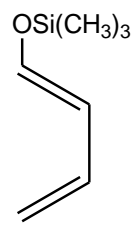

3

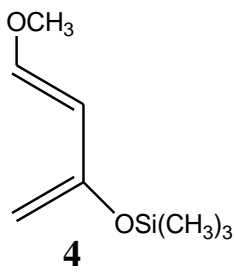

4

Scheme 1

We started the study employing the 2-nitrothiophene 1 with 2 in a sealed ampoule $\left(150^{\circ} \mathrm{C}, 72 \mathrm{~h}\right)$ using benzene as solvent. In this reaction it was observed pyrrolyl-thiophene $\mathbf{5}$ as the principal product formed by an assumed hetero cycloaddition. ${ }^{9}($ Scheme 2 , Table 1 , entry 1 )

On the other hand, the reactions of 1 with 1-trimethylsilyloxy-1,3-butadiene 3 and 1-methoxy-3trimethylsilyloxy-1,3-butadiene ${ }^{9} 4$ under thermal conditions $\left(120^{\circ} \mathrm{C}, 72 \mathrm{~h}\right)$ in benzene as solvent, afforded the benzo[b]thiophen-7-ol 6 and benzo[b]thiophen-5-ol 7, respectively, derived from regioselective cycloaddition to nitrosubstituted bond, with a further aromatization by loss of nitrous acid and methanol (Scheme 2, Table 1, entry 2 and 3). 


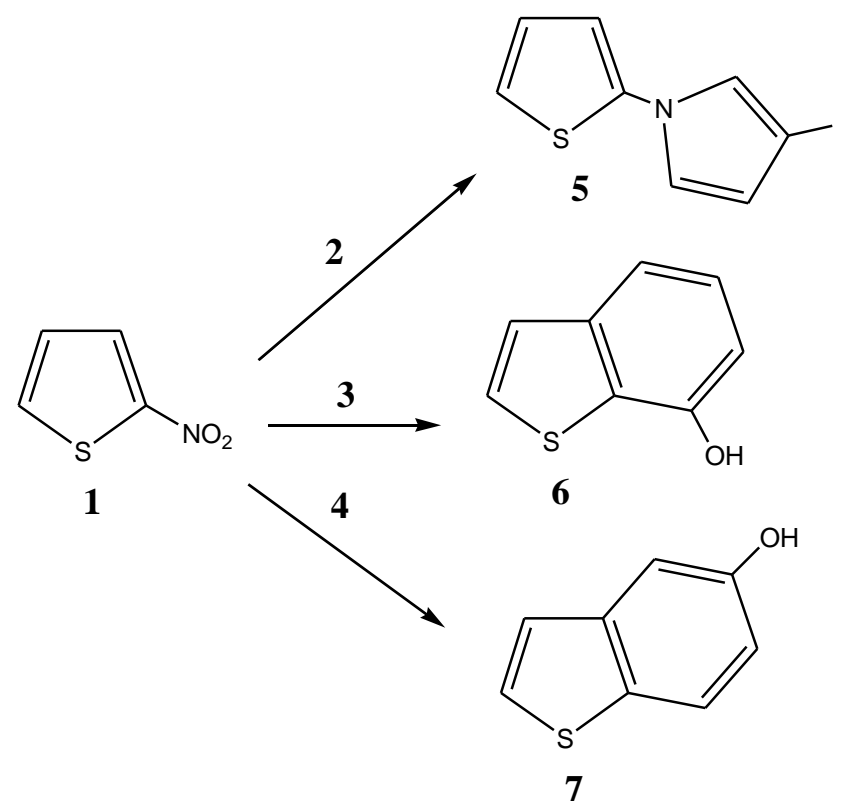

Scheme 2

When the 2-nitrothiophene was reacted with 2 in a sealed ampoule $\left(60^{\circ} \mathrm{C}\right.$ for $\left.24 \mathrm{~h}\right)$ using 1methylimidazolio tetrafluoroborate ([HMIM][BF4]) and ethylammoniun nitrate (EAN) as solvent, the only product observed in a small amount was the pyrrolyl derivative 5 (Table 1 , entry 4 and 5). In contrast to these results, the combination between microwave irradiation with ionic liquids (30 minutes, $180^{\circ} \mathrm{C}$ ) in both cases afforded the same product 5 with better yield hetero DA product (Scheme 2, Table 1, entry 6 and 7). It is necessary taken into account that the normal temperature used in the experiences with MW is $170-180^{\circ} \mathrm{C}$ due to the irradiation of the reaction system need.

Table 1

Diels-Alder reactions of 1 with different dienes

\begin{tabular}{ccccc}
\hline Entry & Diene & Conditions & Product & Yield $^{\mathrm{a}}$ \\
\hline 1 & 2,12 equiv & $150^{\circ} \mathrm{C}^{\mathrm{b}}$ & 5 & traces \\
2 & 3,3 equiv & $120^{\circ} \mathrm{C}^{\mathrm{b}}$ & 6 & 20 \\
3 & 4,3 equiv & $120^{\circ} \mathrm{C}^{\mathrm{b}}$ & 7 & 22 \\
4 & 2,12 equiv & $\mathrm{HMIM}, 60^{\circ} \mathrm{C}^{\mathrm{c}}$ & 5 & traces \\
5 & 2,12 equiv & $\mathrm{EAN}, 60^{\circ} \mathrm{C}^{\mathrm{c}}$ & 5 & traces \\
6 & 2,12 equiv & $\mathrm{HMIM}, 180^{\circ} \mathrm{C}^{\mathrm{d}}$ & 5 & 37 \\
7 & 2,12 equiv & $\mathrm{EAN}, 180^{\circ} \mathrm{C}^{\mathrm{d}}$ & 5 & 40 \\
8 & 2,12 equiv & free solvent, $180^{\circ} \mathrm{C}^{\mathrm{d}}$ & 5 & traces \\
9 & 2,12 equiv & benzene, $180^{\circ} \mathrm{C}^{\mathrm{d}}$ & 5 & 10 \\
\hline
\end{tabular}

${ }^{a}$ Based on consumed dienophile.

${ }^{\mathrm{b}}$ Reactions were carried out for $72 \mathrm{~h}$, benzene as solvent.

${ }^{c}$ Reactions were carried out for $24 \mathrm{~h}$

${ }^{\mathrm{d}}$ Reactions were carried out for 30 minutes and Microwave 
Similar case was observed to make the experience with the same diene-dienophile system, solvent free and microwave irradiation, where product $\mathbf{5}$ is obtained in low yield. (Scheme 2, Table 1, entry 8)

Moreover, the influence of the microwave irradiation was analyzed in the presence of a molecular solvent (benzene) for the reaction of 2-nitrothiophene with isoprene. Again the reaction yielded the $\mathrm{N}$-thiophenpyrrol $\mathbf{5}$ as the principal product. (Scheme 2, Table 1, entry 9).

\section{Conclusion}

In thermal conditions the reactions proceeded in a similar way that when nitrothiophenes are the electrophiles.

On the other hand it was noted that the presence of microwave irradiation in combination with any class of solvent (molecular solvent or protic ILs) improved the yield of the reactions.

This reaction processes should be applicable for the preparation of many biologically interesting molecules.

\section{Acknowledgement}

This research was supported by Argentine Agency of Science and Technology (ANCyT)-PICT 2008 № 1214

\section{References}

1- T. Kokubun, J. Harborne, J. Eagles, P. Waterman. Phytochemistry, Vol. 39, № 5, 1039-1042. (1995).

2- R. Miller, R. Kleiman, R. Powell. Journal of Natural Products, Vol. 51, № 2, 328-330. (1988).

3- M Hussain, N. T. Hung; P. Langer. Tetrahedron Lett. 50, 3929-3932. (2009).

4- (a) Wenkert, E.; Moeller, P.D.M.; Piettre, S. J. Am. Chem. Soc. 1988, 110, 7188-7194; (b) Wenkert, E.; Piettre, S.R. J. Org. Chem, 1988, 53, 5850-5853.

5- C. Della Rosa, M. Kneeteman, P.M.E. Mancini, Tetrahedron Lett. 46, 8711 (2005).

6- C. Della Rosa; M. Kneeteman; P. Mancini, Tetrahedron Lett. 48, 1435-1438 (2007).

7- C. Della Rosa; M. Kneeteman; P. Mancini; Tetrahedron Lett. 48, 7075-7078 (2007).

8- R. Brasca; M. Kneeteman; P. Mancini; W. Fabian, J. Mol. Struct. (THEOCHEM) 911, 124-131 (2009).

9- C. Della Rosa, E. Paredes, M. Kneeteman, P. M. E. Mancini. Letters in Organic Chemistry (LOC) 1, 148-150, (2004). 
10- Thermal reactions. The temperature, the length of the reaction and the diene/dienophile ratio are given in Table 1. An ampoule containing $1.0 \mathrm{mmol}$ of the dienophile and the required amount of diene in $1 \mathrm{ml}$ of ionic liquid was cooled in liquid nitrogen, sealed and then heated with stir in a bath. After the reaction time was completed, it was cooled once more in liquid nitrogen and opened. After separation of the phases, the organic phase was evaporated and the residue purified by column chromatography in silica gel or alumina using hexane/ethyl acetate mixtures as eluent.

Reactions under microwave irradiation. Microwave irradiation was performed in a Anton Paar Monowave 300, microwave reactor in standard closed vessels. 